\title{
ERROR BOUNDS FOR GAUSS-KRONROD QUADRATURE FORMULAE
}

\author{
SVEN EHRICH
}

ABSTRACT. The Gauss-Kronrod quadrature formula $Q_{2 n+1}^{G K}$ is used for a practical estimate of the error $R_{n}^{G}$ of an approxim:ate integration using the Gaussian quadrature formula $Q_{n}^{G}$. Studying an often-used theoretical quality measure, for $Q_{2 n+1}^{G K}$ we prove best presently known bounds for the error constants

$$
c_{s}\left(R_{2 n+1}^{G K}\right)=\sup _{\left\|f^{(s)}\right\|_{\infty} \leq 1}\left|R_{2 n+1}^{G K}[f]\right|
$$

in the case $s=3 n+2+\kappa, \kappa=\left\lfloor\frac{n+1}{2}\right\rfloor-\left\lfloor\frac{n}{2}\right\rfloor$. A comparison with the Gaussian quadrature formula $Q_{2 n+1}^{G}$ shows that there exist quadrature formulae using the same number of nodes but having considerably better error constants.

\section{INTRODUCTION AND STATEMENT OF THE MAIN RESULTS}

For a given nonnegative and integrable weight function $w$ on $[-1,1]$, a quadrature formula $Q_{n}$ and the corresponding remainder $R_{n}$ of (precise) degree of exactness $\operatorname{deg}\left(R_{n}\right)=s$ are linear functionals defined by

$$
\begin{gathered}
Q_{n}[f]=\sum_{\nu=1}^{n} a_{\nu, n} f\left(x_{\nu, n}\right), \quad R_{n}[f]=\int_{-1}^{1} w(x) f(x) d x-Q_{n}[f], \\
\operatorname{deg}\left(R_{n}\right)=s \Leftrightarrow R_{n}\left[p_{\nu}\right]\left\{\begin{array}{ll}
=0, & \nu=0,1, \ldots, s, \\
\neq 0, & \nu=s+1,
\end{array} \quad p_{\nu}(x)=x^{\nu}\right.
\end{gathered}
$$

with nodes $-\infty<x_{1, n}<\cdots<x_{n, n}<\infty$ and weights $a_{\nu, n} \in \mathbb{R}$. It is well known that the Gaussian quadrature formula $Q_{n}^{G}[f]=\sum_{\nu=1}^{n} a_{\nu, n}^{G} f\left(x_{\nu, n}^{G}\right)$ having the highest possible degree of exactness $\operatorname{deg}\left(R_{n}^{G}\right)=2 n-1$ exists uniquely under these assumptions.

In order to obtain an estimate for $R_{n}[f]$ in practice, often a second quadrature formula is used whose nodes, for economical reasons, include $x_{1, n}^{G}, \ldots$, $x_{n, n}^{G}$. If there exist $n+1$ further real and distinct nodes $\xi_{1,2 n+1}, \ldots, \xi_{n+1,2 n+1}$ and weights $\beta_{1,2 n+1}^{(1)}, \ldots, \beta_{n, 2 n+1}^{(1)}, \beta_{1,2 n+1}^{(2)}, \ldots, \beta_{n+1,2 n+1}^{(2)}$ such that the quadrature formula

$$
Q_{2 n+1}^{G K}[f]:=\sum_{\nu=1}^{n} \beta_{\nu, 2 n+1}^{(1)} f\left(x_{\nu, n}^{G}\right)+\sum_{\mu=1}^{n+1} \beta_{\mu, 2 n+1}^{(2)} f\left(\xi_{\mu, 2 n+1}\right)
$$

Received by the editor May 18, 1992 and, in revised form, November 16, 1992.

1991 Mathematics Subject Classification. Primary 65D30; Secondary 41A55.

Key words and phrases. Gauss-Kronrod quadrature formulae, error constants. 
satisfies $\operatorname{deg}\left(R_{2 n+1}^{G K}\right) \geq 3 n+1$, then $Q_{2 n+1}^{G K}$ is called a Gauss-Kronrod quadrature formula. Considering $Q_{2 n+1}^{G K}[f]$ as a much better approximation than $Q_{n}^{G}[f]$, their difference serves as an estimate for $R_{n}^{G}[f]$.

For surveys on this method, cf. Gautschi [5] and Monegato [8], while for existence results with respect to special weight functions $w$ cf., e.g., Szegö [14] and the recent results of Notaris [9] and Peherstorfer [10].

The Gauss-Kronrod method is basic for several practical integration routines, e.g. in QUADPACK [11], and hence one of the most often used methods for approximate integration with practical error estimate. Yet, there is still a need for a theoretical study of $R_{2 n+1}^{G K}$ which could justify the important role GaussKronrod quadrature plays in practical numerical computation (cf. [8, Part II.2]).

As a basis of a systematic study, and as an often-used quality measure, we define the error constants $c_{S}\left(R_{2 n+1}^{G K}\right)$ by

$$
c_{s}\left(R_{2 n+1}^{G K}\right)=\sup _{\left\|f^{(s)}\right\|_{\infty} \leq 1}\left|R_{2 n+1}^{G K}[f]\right|
$$

where $\|g\|_{\infty}:=\sup _{x \in[-1,1]}|g(x)|$ for $g:[-1,1] \rightarrow \mathbb{R}$. By definition, the error constants $c_{s}\left(R_{2 n+1}^{G K}\right)$ are the smallest real values independent of $f$ satisfying the standard error bounds

$$
\left|R_{2 n+1}^{G K}[f]\right| \leq c_{s}\left(R_{2 n+1}^{G K}\right)\left\|f^{(s)}\right\|_{\infty}
$$

and are well known to exist whenever $\operatorname{deg}\left(R_{2 n+1}^{G K}\right) \geq s-1$ (cf. [1]).

Brass and Förster [2] proved

$$
\frac{c_{2 n}\left(R_{2 n+1}^{G K}\right)}{c_{2 n}\left(R_{n}^{G}\right)} \leq \mathrm{const} \sqrt[4]{n}\left(\frac{1}{3.493 \ldots}\right)^{n}
$$

and considered this result as a theoretical explanation for the significant superiority of $Q_{2 n+1}^{G K}$ over $Q_{n}^{G}$.

Only very little is known about the quality of $Q_{2 n+1}^{G K}$ itself. Rabinowitz [12] proved the existence of $c_{s}\left(R_{2 n+1}^{G K}\right)$ for $s=1, \ldots, 3 n+2+\kappa, \kappa=\left\lfloor\frac{n+1}{2}\right\rfloor-\left\lfloor\frac{n}{2}\right\rfloor$ and nonexistence for $s>3 n+2+\kappa$, showing that $\operatorname{deg}\left(R_{2 n+1}^{G K}\right)=3 n+1+\kappa$; cf. also Rabinowitz [13] for a proof of the nondefiniteness of $R_{2 n+1}^{G K}$. Brass and Förster [2], Brass and Schmeisser [3], and Monegato [7] proved upper bounds for $c_{3 n+2+\kappa}\left(R_{2 n+1}^{G K}\right)$. In the following theorem, we give lower bounds as well as new upper bounds for $c_{3 n+2+\kappa}\left(R_{2 n+1}^{G K}\right)$ in the case $w \equiv 1$ that improve the hitherto best-known bounds.

Theorem. Let $w \equiv 1, n \geq 4, \kappa=\left\lfloor\frac{n+1}{2}\right\rfloor-\left\lfloor\frac{n}{2}\right\rfloor$. Then there holds

$$
c_{3 n+2+\kappa}\left(R_{2 n+1}^{G K}\right) \leq \bar{c}_{3 n+2+\kappa}\left(R_{2 n+1}^{G K}\right),
$$

where

$$
\begin{aligned}
\bar{c}_{3 n+2+\kappa}\left(R_{2 n+1}^{G K}\right):=\frac{1}{(3 n+2+\kappa) ! 2^{3 n+\kappa}}\{ & \frac{17}{10 \sqrt{3 n-3(2+\kappa)}} \\
& \left.+\frac{2 \sqrt{2}}{\sqrt{(6 n+3+2 \kappa)(6 n+5+2 \kappa)}}\right\} .
\end{aligned}
$$


Furthermore, for $n$ even there holds

$$
\begin{aligned}
c_{3 n+2}\left(R_{2 n+1}^{G K}\right) \geq & \frac{1}{3 e \sqrt{\pi}} \frac{2^{n+3}}{(3 n+2) !} \frac{[n !]^{4}}{(2 n) !(2 n+1) !} \\
& \cdot \sqrt{\frac{3 n-2}{n^{2}-2 n}} \frac{(2 n+3)(5 n-3)^{2}}{(n-2)^{2}(n+2)(3 n-1)^{3}(3 n+1)^{2}},
\end{aligned}
$$

while for $n$ odd there holds

$$
\begin{aligned}
c_{3 n+3}\left(R_{2 n+1}^{G K}\right) \geq & \frac{1}{27 e \sqrt{\pi}} \frac{2^{n+3}}{(3 n+3) !} \frac{[n !]^{4}}{(2 n) !(2 n+1) !} \\
& \cdot \sqrt{\frac{3 n-1}{n^{2}-3 n}} \frac{(2 n+3)\left(5 n^{2}-7 n+3\right)^{2}}{(n-1)^{2} n^{2}(n+2)(n-3)^{2}(3 n-3)(3 n-2)^{2}} .
\end{aligned}
$$

Remark. While the upper bound in the theorem improves known results, it may still be sharpened. However, an improvement can only be obtained by a polynomial factor, since it follows that

$$
\frac{\bar{c}_{3 n+2+\kappa}\left(R_{2 n+1}^{G K}\right)}{c_{3 n+2+\kappa}\left(R_{2 n+1}^{G K}\right)}=O\left(n^{5}\right)
$$

if we replace $c_{3 n+2+\kappa}\left(R_{2 n+1}^{G K}\right)$ by the lower bounds (1). A result of Brass and Schmeisser [3, Theorem 7] implies that amongst all quadrature formulae $Q_{m}$ with positive weights and degree of exactness $\operatorname{deg}\left(Q_{m}\right) \geq 3 n+1+\kappa$, the Lobatto formula $Q_{m^{*}}^{L_{0}}$ for $m^{*}=\frac{3 n+2+\kappa}{2}$ is worst with respect to the error constant $c_{3 n+2+\kappa}\left(R_{2 n+1}^{G K}\right)$, i.e. (cf. [1, p. 149])

$$
c_{3 n+2+\kappa}\left(R_{m}\right) \leq c_{3 n+2+\kappa}\left(R_{m^{*}}^{L_{0}}\right)=\pi 2^{-(3 n+2+\kappa)}(1+o(1)) \text { as } n \rightarrow \infty .
$$

The lower bounds (1) now prove that $Q_{2 n+1}^{G K}$ can be better than this upper bound only by a polynomial factor $\left(O\left(n^{5.5}\right)\right)^{-1}$. Note that Brass and Schmeisser [3, Remark 4] explicitly construct a positive quadrature formula $Q_{3 n+2+\kappa}^{B S}$ that satisfies

$$
c_{3 n+2+\kappa}\left(R_{3 n+2+\kappa}^{B S}\right)=\pi 4^{-(3 n+2+\kappa)}(1+o(1)) \text { as } n \rightarrow \infty .
$$

In the following corollary we will compare $Q_{2 n+1}^{G K}$ and $Q_{2 n+1}^{G}$ in order to show that there also exist quadrature formulae using the same number of nodes but having considerably better error constants.

Corollary. Let $w \equiv 1, n \geq 1$. Then $c_{3 n+2+\kappa}\left(R_{2 n+1}^{G}\right) \leq c_{3 n+2+\kappa}\left(R_{2 n+1}^{G K}\right)$, and we have equality only in the case $n=1$, where the Gaussian formula and the Gauss-Kronrod formula are identical. Furthermore, for $n \geq 15$ there holds the sharper bound

$$
\frac{c_{3 n+2+\kappa}\left(R_{2 n+1}^{G}\right)}{c_{3 n+2+\kappa}\left(R_{2 n+1}^{G K}\right)} \leq 3^{-n+1}
$$

while asymptotically we find

$$
\lim _{n \rightarrow \infty} \sqrt[n]{\frac{c_{3 n+2+\kappa}\left(R_{2 n+1}^{G}\right)}{c_{3 n+2+\kappa}\left(R_{2 n+1}^{G K}\right)}}=\sqrt{\frac{6^{6}}{7^{7}}}=\frac{1}{4.2013 \ldots} .
$$




\section{Proofs of the Results}

In the sequel let $m=\left\lfloor\frac{n+1}{2}\right\rfloor, \kappa=m-\left\lfloor\frac{n}{2}\right\rfloor$, and let $\mathscr{P}_{s}$ denote the space of polynomials of degree less than or equal to $S$.

Proof of the Theorem. We will first prove the lower bounds (1). Let $E_{n+1} \in \mathscr{P}_{n+1}$ be defined by

$$
E_{n+1}=\sum_{\nu=0}^{m-1} \alpha_{\nu, n} T_{n+1-2 \nu}+\left\{\begin{array}{lc}
\alpha_{m, n} T_{1}, & n \text { even } \\
\frac{1}{2} \alpha_{m, n}, & n \text { odd }
\end{array}\right.
$$

where $T_{\nu}$ denotes the $\nu$ th Chebyshev Polynomial of the first kind and

$$
\begin{gathered}
\alpha_{0, n}=1, \quad \alpha_{\nu, n}=-f_{\nu, n}-\sum_{\mu=1}^{\nu-1} f_{\mu, n} \alpha_{\nu-\mu, n}, \quad \nu=2,3, \ldots \\
f_{0, n}=1, \quad f_{\nu, n}=\sigma_{\nu, n} f_{\nu-1, n}, \quad \sigma_{\nu, n}=\left(1-\frac{1}{2 \nu}\right)\left(1-\frac{1}{2 n+2 \nu+1}\right) .
\end{gathered}
$$

For reasons of simplicity, when no ambiguity arises, we do not indicate the dependence on $n$, i.e., $\alpha_{\nu}:=\alpha_{\nu, n}, f_{\nu}:=f_{\nu, n}$, and $\sigma_{\nu}:=\sigma_{\nu, n}$. The zeros of $E_{n+1}$ are the additionally chosen nodes $\xi_{1,2 n+1}, \ldots, \xi_{n+1,2 n+1}$ of $Q_{2 n+1}^{G K}$ (cf. [7]). Rabinowitz [12] showed that with $g_{\kappa}=P_{n} E_{n+1} P_{n+1+\kappa} \quad\left(P_{\nu}\right.$ denoting the $\nu$ th Legendre Polynomial) there holds, for $n$ even,

$$
R_{2 n+1}^{G K}\left[g_{0}\right]=\frac{1}{2 n+2}\left(\alpha_{m}-\alpha_{m+1}\right)
$$

while for $n$ odd, there holds

$$
R_{2 n+1}^{G K}\left[g_{1}\right]=\frac{2 n+3}{(2 n+2)(2 n+4)}\left(\alpha_{m-1}-\alpha_{m+1}\right) .
$$

From the definition of the error constants $c_{s}\left(R_{2 n+1}^{G K}\right)$ we get

$$
c_{s}\left(R_{2 n+1}^{G K}\right) \geq \frac{\left|R_{2 n+1}^{G K}[f]\right|}{\left\|f^{(s)}\right\|_{\infty}} \text { for all } f \in C^{s}[-1,1] \backslash \mathscr{P}_{s-1} .
$$

Defining $p_{\nu}(x):=x^{\nu}$, we conclude

$$
c_{3 n+2+\kappa}\left(R_{2 n+1}^{G K}\right) \geq \frac{\left|R_{2 n+1}^{G K}\left[p_{3 n+2+\kappa}\right]\right|}{(3 n+2+\kappa) !} .
$$

Taking into account the leading coefficients of $P_{\nu}$ and $E_{n+1}$, one readily verifies

$$
g_{\kappa}(x)=\frac{(2 n) !}{2^{n}(n !)^{2}} 2^{n} \frac{(2 n+2+2 \kappa) !}{2^{n+1+\kappa}[(n+1+\kappa) !]^{2}} x^{3 n+2+\kappa}+p(x), \quad p \in \mathscr{P}_{3 n+1+\kappa}
$$

and, using the linearity and the degree of exactness of $R_{2 n+1}^{G K}$,

$$
R_{2 n+1}^{G K}\left[p_{3 n+2+\kappa}\right]=\frac{2^{n-1-\kappa}[n !]^{4}}{(2 n) !(2 n+1) !}\left(\alpha_{m-\kappa}-\alpha_{m+1}\right)
$$

We will now derive lower bounds for the difference $\left|\alpha_{m-\kappa}-\alpha_{m+1}\right|$. Szegö [14] proved that the sequence $\left(-\alpha_{\nu+1}\right)$ is positive throughout, and completely monotonic, i.e.,

$$
(-1)^{\nu+1} \Delta^{\nu} \alpha_{\nu+1}>0, \quad \nu=0,1,2, \ldots,
$$

while for its sum he proved $\sum_{\nu=0}^{\infty} \alpha_{\nu+1}=-1$. In the following Lemma 1 we state some further properties of $\left(\alpha_{\nu}\right)$ needed here; the proof of Lemma 1 will be given later. 
Lemma 1. The sequence $\left(\alpha_{\nu}\right)$ satisfies

$$
\alpha_{0}=1, \quad \alpha_{1}=-f_{1}, \quad \alpha_{\nu}=\sum_{\mu=1}^{\nu-1} \alpha_{\mu} f_{\nu-1-\mu}\left(\sigma_{\nu}-\sigma_{\nu-\mu}\right), \nu \geq 2 .
$$

Bounds for $\left(\alpha_{\nu}\right)$ are given by

$$
f_{\nu-1}\left(\sigma_{\nu}-\sigma_{\nu-1}\right)<\left|\alpha_{\nu}\right|<f_{\nu-1}\left(\sigma_{\nu}-\sigma_{1}\right) .
$$

Lower bounds for the differences of $\left(\alpha_{\nu}\right)$ are, for every $p \in \mathbb{N}$,

$$
\begin{gathered}
p\left(\alpha_{\nu+1}-\alpha_{\nu}\right) \geq \alpha_{\nu+p}-\alpha_{\nu}, \\
p\left(\alpha_{\nu+1}-\alpha_{\nu-1}\right) \geq \alpha_{\nu+2 p+1}-\alpha_{\nu-1} .
\end{gathered}
$$

According to the lower bounds for the differences in Lemma 1, we are now looking for $p=p(n)$ such that the right-hand sides of

$$
\left|\alpha_{m}-\alpha_{m+1}\right| \geq \frac{1}{p(n)}\left(f_{m-1}\left(\sigma_{m}-\sigma_{m-1}\right)-f_{m+p(n)-1}\left(1-\sigma_{1}\right)\right)
$$

for $n$ even, and

$$
\left|\alpha_{m-1}-\alpha_{m+1}\right| \geq \frac{1}{p(n)}\left(f_{m-2}\left(\sigma_{m-1}-\sigma_{m-2}\right)-f_{m+2 p(n)}\left(1-\sigma_{1}\right)\right)
$$

for $n$ odd, be positive and as big as possible. (Recall the definition of $m$ at the beginning of this section.) By (2) we calculate $1-\sigma_{1}=\frac{n+2}{2 n+3}$, and for even $n>2$,

$$
\sigma_{m}-\sigma_{m-1}=\frac{4(5 n-3)}{(n-2)(3 n-1)(3 n+1)}
$$

while for odd $n>3$,

$$
\sigma_{m-1}-\sigma_{m-2}=\frac{4\left(5 n^{2}-7 n+3\right)}{3 n(n-3)(n-1)(3 n-2)}
$$

Now let $n$ be even. The following representation of the $f_{\nu}$ can easily be proved by induction:

$$
f_{\nu}=\frac{(2 \nu) !}{[\nu !]^{2}}\left[\frac{(n+\nu) !}{n !}\right]^{2} \frac{(2 n+1) !}{(2 n+2 \nu+1) !} .
$$

We determine $p=p(n)$ such that

$$
f_{m-1} \frac{4(5 n-3)}{(n-2)(3 n-1)(3 n+1)}-f_{m+p(n)-1} \frac{n+2}{2 n+3}>0
$$

holds, which is equivalent to

$$
\begin{aligned}
\frac{4(2 n+3)(5 n-3)}{(n-2)(n+2)(3 n-1)(3 n+1)} \\
>\frac{(2 m+2 p(n)-2) !}{[(m+p(n)-1) !]^{2}} \frac{((m-1) !)^{2}}{(2 m-2) !} \frac{(2 n+2 m-2) !}{((n+m-1) !)^{2}} \\
\quad \cdot \frac{((n+m+p(n)-1) !)^{2}}{(2 n+2 m+2 p(n)-2) !}
\end{aligned}
$$


For the right side of the inequality we find by some elementary calculation, using Stirling's formula,

$$
\begin{aligned}
& \frac{(2 m+2 p(n)-2) !}{[(m+p(n)-1) !]^{2}} \frac{((m-1) !)^{2}}{(2 m-2) !} \frac{(2 n+2 m-2) !}{((n+m-1) !)^{2}} \frac{((n+m+p(n)-1) !)^{2}}{(2 n+2 m+2 p(n)-2) !} \\
& \quad<\sqrt{e} \frac{2 n+2 m-1}{2 n+2 m+2 p(n)-1} .
\end{aligned}
$$

Replacing the right side of (5) by this simpler estimate, the following condition for $p(n)$ arises:

$$
2 p(n) \geq \frac{\sqrt{e}(n-2)(n+2)(3 n-1)^{2}(3 n+1)}{4(5 n-3)(2 n+3)}-(3 n-1) .
$$

We may now choose $p(n)$ for $n$ even as

$$
p(n):=\left\lceil c \cdot \frac{\sqrt{e}(n-2)(n+2)(3 n-1)^{2}(3 n+1)}{8(2 n+3)(5 n-3)}-\frac{3 n-1}{2}\right\rceil, \quad c \geq 1,
$$

leading to

$$
\left|\alpha_{m}-\alpha_{m+1}\right|>\frac{1}{p(n)} f_{m-1} \frac{4(5 n-3)}{(n-2)(3 n-1)(3 n+1)}\left(1-\frac{1}{c}\right),
$$

where for $n \geq 4$ from (4), using Stirling's formula, we can show

$$
f_{m-1} \geq \frac{2}{3 \sqrt{e \pi}} \sqrt{\frac{3 n-2}{n(n-2)}}
$$

Since by definition of $p(n)$,

$$
p(n) \leq c \cdot \frac{\sqrt{e}(n-2)(n+2)(3 n-1)^{2}(3 n+1)}{8(5 n-3)(2 n+3)},
$$

we readily conclude

$$
\left|\alpha_{m}-\alpha_{m+1}\right|>\frac{64}{3 e \sqrt{\pi}} \sqrt{\frac{3 n-2}{n(n-2)}} \frac{(2 n+3)(5 n-3)^{2}}{(n-2)(n+2)(3 n-1)^{3}(3 n+1)^{2}}\left(\frac{c-1}{c^{2}}\right) .
$$

Maximizing the right side with respect to $c \geq 1$ leads to $c=2$ and therefore to the asserted inequality for $n$ even. For the proof of the lower bound for $\left|\alpha_{m-1}-\alpha_{m+1}\right|, n$ odd, we can proceed in an analogous way. This time, a sufficient condition for the $p(n)$ is

$$
\frac{4(2 n+3)\left(5 n^{2}-7 n+3\right)}{3(n-3)(n-1) n(n+2)(3 n-2)}>\sqrt{e} \frac{2 n+2 m-4}{2 n+2 m+4 p(n)+1} .
$$

For $n$ odd, we then choose

$p(n):=\left\lceil c \cdot \frac{3 \sqrt{e}(n-3)(n-1) n(n+2)(3 n-3)(3 n-2)}{16(2 n+3)\left(5 n^{2}-7 n+3\right)}-\frac{3 n+2}{4}\right\rceil, \quad c \geq 1$.

Using the inequality

$$
f_{m-2} \geq \frac{2}{3 \sqrt{e \pi}} \sqrt{\frac{3 n-1}{n(n-3)}}
$$

leads again, after maximization with respect to $c$, to the lower bound for $n$ odd.

We will now prove the upper bounds stated in the Theorem with the help of the following lemma, which is contained in [2, Theorem 1]. 
Lemma 2. Let $R$ be a continuous, linear functional on $C[-1,1]$ satisfying $R\left[\mathscr{P}_{m-1}\right]=0$, and let

$$
K_{m, s}(x):=\frac{2}{\pi} \frac{m ! 2^{m}}{(2 m) !} \sum_{\mu=0}^{s-1}\left(1-x^{2}\right)^{m-1 / 2} R\left[T_{m+\mu}\right] \frac{P_{\mu}^{(m)}(x)}{P_{\mu}^{(m)}(1)},
$$

where $P_{\mu}^{(m)}$ denotes the $\mu$ th ultraspherical polynomial of order $m$. The limit

$$
K_{m}(x):=\lim _{s \rightarrow \infty} K_{m, s}(x), \quad x \in[-1,1],
$$

exists. If $f \in C^{m}[-1,1]$, then

$$
R[f]=\int_{-1}^{1} f^{(m)}(x) K_{m}(x) d x .
$$

If $s=0,1,2, \ldots$, then

$$
c_{m}(R)=\int_{-1}^{1}\left|K_{m, s}(x)\right| d x+\rho_{s}
$$

where

$$
\left|\rho_{s}\right| \leq \frac{1}{m ! 2^{m-1}}\left\{\frac{(2 m) ! s !}{(2 m+s) !} \frac{2 m+s}{2 m-1}\right\}^{1 / 2} \sup _{\mu \geq s}\left|R\left[T_{\mu+m}\right]\right| .
$$

Using (3) and $T_{\nu}(x)=2^{\nu-1} x^{\nu}+p(x), p \in \mathscr{P}_{\nu-1}$, we find

$$
R_{2 n+1}^{G K}\left[T_{3 n+2+\kappa}\right]=\frac{2^{4 n}[n !]^{4}}{(2 n) !(2 n+1) !}\left(\alpha_{m-\kappa}-\alpha_{m+1}\right) .
$$

Taking advantage of the monotonicity of $\left(-\alpha_{\nu+1}\right)$, from Lemma 1 we get

$$
\left|\alpha_{m-\kappa}-\alpha_{m+1}\right|<\left|\alpha_{m-\kappa}\right|<f_{m-1-\kappa}\left(\sigma_{m-\kappa}-\sigma_{1}\right) \text {. }
$$

Using (4), we obtain by the use of Stirling's formula

$$
f_{m-1-\kappa} \leq \frac{2 n+2-\kappa}{3 n} \sqrt{\frac{3 n-2+\kappa}{n(n-2-\kappa)}} .
$$

According to (2) we get for $n$ even

$$
\sigma_{m}-\sigma_{1}=\frac{3 n^{2}-n-10}{(2 n+3)(3 n+1)}
$$

and for $n$ odd

$$
\sigma_{m-1}-\sigma_{1}=\frac{3 n^{3}-5 n^{2}-14 n+6}{(n-1)(2 n+3)(3 n-2)} .
$$

Lemma 2 implies for $R=R_{2 n+1}^{G K}, s=1$, and $m=3 n+2+\kappa$

$$
\begin{aligned}
c_{3 n+2+\kappa} & \left(R_{2 n+1}^{G K}\right) \\
\leq & \left|R_{2 n+1}^{G K}\left[T_{3 n+2+\kappa}\right]\right| \int_{-1}^{1}\left|\frac{2}{\pi} \frac{(3 n+2+\kappa) ! 2^{3 n+2+\kappa}}{(6 n+4+2 \kappa) !}\left(1-x^{2}\right)^{3 n+2+\kappa-1 / 2}\right| d x \\
& +\frac{1}{(3 n+2+\kappa) ! 2^{3 n+1+\kappa}}\left\{\frac{2(6 n+4+2 \kappa) !}{(6 n+6+2 \kappa) !} \frac{6 n+6+2 \kappa}{6 n+3+2 \kappa}\right\}^{1 / 2} \\
& \cdot \sup _{\mu \geq 2}\left|R_{2 n+1}^{G K}\left[T_{3 n+2+\kappa+\mu}\right]\right| .
\end{aligned}
$$


Since

$$
\int_{-1}^{1}\left|\left(1-x^{2}\right)^{3 n+2+\kappa-1 / 2}\right| d x=\frac{\Gamma\left(\frac{1}{2}\right) \Gamma\left(3 n+2+\kappa+\frac{1}{2}\right)}{\Gamma(3 n+3+\kappa)}
$$

and $\sup _{\mu \geq 2}\left|R_{2 n+1}^{G K}\left[T_{3 n+2+\mu}\right]\right| \leq 4$, we find

$$
\begin{aligned}
c_{3 n+2+\kappa}\left(R_{2 n+1}^{G K}\right) \leq & \frac{1}{(3 n+2+\kappa) ! 2^{3 n+1+\kappa}} \\
& \cdot\left(\left|R_{2 n+1}^{G K}\left[T_{3 n+2+\kappa}\right]\right|+\frac{4 \sqrt{2}}{\sqrt{(6 n+3+2 \kappa)(6 n+5+2 \kappa)}}\right) .
\end{aligned}
$$

Using (6), (7), (8), (9), and (10), we conclude the result after some simplifying calculation.

Proof of Lemma 1. The recursion formula (2) for $\alpha_{\nu}$ and $\alpha_{\nu-1}$, respectively, is

$$
\alpha_{\nu}=-f_{\nu}-\sum_{\mu=1}^{\nu-1} \alpha_{\mu} f_{\nu-\mu}, \quad 0=-f_{\nu-1}-\sum_{\mu=1}^{\nu-1} \alpha_{\mu} f_{\nu-1-\mu} .
$$

Multiplication of the first by $f_{\nu-1}$ and the second by $-f_{\nu}$, and summation, yields

$$
\begin{aligned}
f_{\nu-1} \alpha_{\nu} & =\sum_{\mu=1}^{\nu-1} \alpha_{\mu}\left[f_{\nu-1-\mu} f_{\nu}-f_{\nu-\mu} f_{\nu-1}\right]=\sum_{\mu=1}^{\nu-1} \alpha_{\mu} f_{\nu-1} f_{\nu-1-\mu}\left(\sigma_{\nu}-\sigma_{\nu-\mu}\right) \\
& =f_{\nu-1} \sum_{\mu=1}^{\nu-1} \alpha_{\mu} f_{\nu-1-\mu}\left(\sigma_{\nu}-\sigma_{\nu-\mu}\right),
\end{aligned}
$$

leading to the first assertion (see also [6] for this method). Using $f_{\nu}>0$ and $\sigma_{\nu}-\sigma_{\nu-\mu}>0$ for $\mu=1, \ldots, \nu-1$, we find that all terms in the righthand sum have the same sign, and we conclude the second assertion, using the monotonicity of $\left(\sigma_{\nu}\right)$. Since $\left(-\alpha_{\nu+1}\right)$ is completely monotonic, the last two inequalities of Lemma 1 follow readily.

Proof of the Corollary. Theorem 4 of [2] states that

$$
c_{3 n+2+\kappa}\left(R_{2 n+1}^{G}\right) \leq \bar{c}_{3 n+2+\kappa}\left(R_{2 n+1}^{G}\right),
$$

where

$$
\begin{aligned}
\bar{c}_{3 n+2+\kappa}\left(R_{2 n+1}^{G}\right):= & \frac{\pi}{(3 n+2+\kappa) ! 2^{3 n+2+\kappa}} \\
& \cdot\left\{\frac{(n-\kappa) !(6 n+4+2 \kappa) !}{(7 n+4+\kappa) !} \frac{7 n+4+\kappa}{8 n+4}\right. \\
& \left.\cdot\left(1+\frac{5}{3} \frac{(n+1-\kappa)(n+2-\kappa)}{(6 n+3+2 \kappa)(7 n+5+\kappa)}\right)\right\}^{1 / 2} .
\end{aligned}
$$

Using the lower bounds (1) for $c_{3 n+2+\kappa}\left(R_{2 n+1}^{G K}\right)$, the following inequality can be proved for $n \geq 4$ after some (elementary) calculation involving Stirling's formula:

$$
\frac{c_{3 n+2+\kappa}\left(R_{2 n+1}^{G}\right)}{c_{3 n+2+\kappa}\left(R_{2 n+1}^{G K}\right)}<c \cdot n^{23 / 4}\left(\sqrt{\frac{6^{6}}{7^{7}}}\right)^{n}
$$


with $c=0.62$. Inequality (11) yields $c_{3 n+2+\kappa}\left(R_{2 n+1}^{G}\right) \leq c_{3 n+2+\kappa}\left(R_{2 n+1}^{G K}\right)$ for $n \geq 10$ and $c_{3 n+2+\kappa}\left(R_{2 n+1}^{G}\right) / c_{3 n+2+\kappa}\left(R_{2 n+1}^{G K}\right) \leq 3^{-n+1}$ for $n \geq 68$, where for $1 \leq n \leq 9$ and $15 \leq n \leq 68$, respectively, the results can be proved numerically (cf. [4]). In [2, Theorem 4] it is stated that the upper bound $\bar{c}_{3 n+2+\kappa}\left(R_{2 n+1}^{G}\right)$ can only be improved by $O\left(n^{3 / 4}\right)$. Using this result and the remark in $\S 1$ to obtain also a lower bound for the ratio in (11), the corollary follows.

\section{FURTHER REMARKS}

1. Using similar methods as described above, respective results can be obtained in the more general case of an ultraspherical weight function (cf. [4]). For $w(x)=\left(1-x^{2}\right)^{\lambda-1 / 2}, \lambda \in(0,1), n \geq 4, \kappa=\left\lfloor\frac{n+1}{2}\right\rfloor-\left\lfloor\frac{n}{2}\right\rfloor$ there holds $c_{3 n+2+\kappa}\left(R_{2 n+1}^{G K}\right) \leq \bar{c}_{3 n+2+\kappa}\left(R_{2 n+1}^{G K}\right)$, where

$$
\begin{aligned}
& \bar{c}_{3 n+2+\kappa}\left(R_{2 n+1}^{G K}\right) \\
&:=\frac{1}{(3 n+2+\kappa) ! 2^{3 n+1+\kappa}}\left(\frac{\pi \lambda}{2^{2 \lambda-1} \Gamma(1-\lambda)} \frac{9 n+4 \lambda}{9 n-3 \kappa+4 \lambda}\right. \\
& \cdot\left\{\frac{6(2 n+2+\lambda)}{(n+\lambda-1+\kappa)(9 n+4 \lambda)}\right\}^{\lambda} \\
&\left.+\frac{2 \sqrt{\pi}(\lambda+(1+\kappa) / 4)^{\lambda-1}}{\sqrt{(6 n+3+2 \kappa)(6 n+5+2 \kappa)}}\right) .
\end{aligned}
$$

Lower bounds for $c_{3 n+2+\kappa}\left(R_{2 n+1}^{G K}\right)$ can be found in [4], which for the quality of the upper bounds yield

$$
\frac{\bar{c}_{3 n+2+\kappa}\left(R_{2 n+1}^{G K}\right)}{c_{3 n+2+\kappa}\left(R_{2 n+1}^{G K}\right)}=O\left(n^{3+1 / \lambda}\right)
$$

The corresponding error constants of $Q_{2 n+1}^{G}$ are again significantly smaller (cf. [4]).

2. Using the methods derived in the proof of the theorem in $\S 1$, we can also prove bounds for the error constants $c_{3 n+2+\kappa-s}\left(R_{2 n+1}^{G K}\right)$ of nonmaximum order (cf. [4] for details). In the case of constant $s \in \mathbb{N}$, these bounds are again better than the hitherto best-known bounds. For their quality we can prove

$$
\frac{\bar{c}_{3 n+2+\kappa-s}\left(R_{2 n+1}^{G K}\right)}{c_{3 n+2+\kappa-s}\left(R_{2 n+1}^{G K}\right)}=O\left(n^{5+(s / 2)}\right) \text {. }
$$

In the case $s=s(n) \in \mathbb{N}, \lim _{n \rightarrow \infty} \frac{3 n+2+\kappa-s}{n}=A>0$, the new bounds are of quality

$$
\lim _{n \rightarrow \infty} \sqrt[n]{\frac{\bar{c}_{3 n+2+\kappa-s}\left(R_{2 n+1}^{G K}\right)}{c_{3 n+2+\kappa-s}\left(R_{2 n+1}^{G K}\right)}}=\frac{A^{A}(3-A)^{(3-A) / 2}(3+A)^{(3+A) / 2}}{3^{3}(2 A)^{A}} .
$$

The corresponding error constants of $Q_{2 n+1}^{G}$ can be proved to be significantly smaller for $2<A \leq 3$.

\section{ACKNOWLEDGMENT}

I would like to thank the referee for the helpful comments concerning the presentation of this paper. 


\section{BIBLIOGRAPHY}

1. H. Brass, Quadraturverfahren, Vandenhoeck und Ruprecht, Göttingen, 1977.

2. H. Brass and K.-J. Förster, On the estimation of linear functionals, Analysis 7 (1987), 237-258.

3. H. Brass and G. Schmeisser, Error estimates for interpolatory quadrature formulae, Numer. Math. 37 (1981), 371-386.

4. S. Ehrich, Error estimates for Gauss-Kronrod quadrature formulae, Hildesheimer Informatik-Berichte 14 (1991).

5. W. Gautschi, Gauss-Kronrod quadrature-A survey, Numerical Methods of Approximation Theory. III (G. V. Milovanović, ed.), University of Niš, 1988, pp. 39-66.

6. T. Kaluza, Über die Koeffizienten reziproker Potenzreihen, Math. Z. 28 (1928), 161-170.

7. G. Monegato, Some remarks on the construction of extended Gaussian quadrature rules, Math. Comp. 32 (1978), 247-252.

8. __ Stieltjes polynomials and related quadrature rules, SIAM Rev. 24 (1982), 137-158.

9. S. E. Notaris, Gauss-Kronrod quadrature formulae for weight functions of Bernstein-Szegö type. II, J. Comput. Appl. Math. 29 (1990), 161-169.

10. F. Peherstorfer, Weight functions admitting repeated positive Kronrod quadrature, BIT 30 (1990), 145-151.

11. R. Piessens, E. de Doncker, C. Überhuber, and D. K. Kahaner, QUADPACK-A subroutine package for automatic integration, Springer Ser. in Comput. Math., vol. 1, Springer-Verlag, Berlin, 1983.

12. P. Rabinowitz, The exact degree of precision of generalized Gauss-Kronrod integration rules, Math. Comp. 35 (1980), 1275-1283.

13. _ On the definiteness of Gauss-Kronrod integration rules, Math. Comp. 46 (1986), 225-227.

14. G. Szegö, Über gewisse orthogonale Polynome, die zu einer oszillierenden Belegungsfunktion gehören, Math. Ann. 110 (1934), 501-513.

Universität Hildesheim, Institut für Mathematik, Marienburger Platz 22, D-31141 HiLDESHEIM, GERMANY

E-mail address: ehrich@mathematik.uni-hildesheim.de 